\title{
Synthesis of submicron barium carbonate using a high-gravity technique
}

\author{
Clifford Y. Tai*, Chia-te Tai, Hwai-shen Liu \\ Department of Chemical Engineering, National Taiwan University, Taipei 106, Taiwan
}

Received 19 May 2006; received in revised form 24 August 2006; accepted 24 August 2006

Available online 14 September 2006

\begin{abstract}
The purpose of this study was to build a platform for producing fine particles by applying a high-gravity (higee) technique to achieve reactive precipitation. Barium carbonate was chosen as a model compound and was produced in a spinning disk reactor (SDR), which is one type of higee equipment, via a carbonation route and a once-through mode. For size measurement, a suitable dispersion method was developed to obtain reproducible particle size data, using a laser-light analyzer. Several factors that affected the particle size of barium carbonate, including the $\mathrm{CO}_{2}$ flow rate, the feed rate of $\mathrm{Ba}(\mathrm{OH})_{2}$ slurry, the rotation speed, and the solid-content of feed slurry, were investigated. A high rotating speed and low feeding rate of slurry yielded small particles. The optimum solid-content of feeding slurry for obtaining small particles was also determined. However, the effect of the $\mathrm{CO}_{2}$ flow rate on the particle size of the product was not significant.
\end{abstract}

(C) 2006 Elsevier Ltd. All rights reserved.

Keywords: High-gravity (higee) field; Fine particles; Spinning disk reactor; Barium carbonate; Precipitation; Process intensification

\section{Introduction}

Synthetic barium carbonate under ambient conditions appears in a form of white powder and decomposes to barium oxide and carbon dioxide at about $1300^{\circ} \mathrm{C}$. There are three polymorphisms of $\mathrm{BaCO}_{3}$, i.e., orthorhombic, hexagonal, and cubic, but only the orthorhombic phase is obtained under ambient conditions. As far as the crystal habits are concerned, rod-like, needle-like, and olivary barium carbonates are mostly found (Yagi et al., 1988; Kubota et al., 1990; Chen et al., 2001). Rod-like and needle-like habits have been obtained via a carbonation route at ambient temperatures. Barium carbonate is commonly used as a raw material for synthesizing other ceramics, such as barium titanate, of electronic and magnetic materials. Recently, a report by Tagaya et al. (2003) indicated that birefringence of an optical polymer film could be eliminated during the drawing step in polymer processing by doping rod-like inorganic particles, which were below $400 \mathrm{~nm}$ in size. Therefore, the commercial value of $\mathrm{BaCO}_{3}$ powders may be significant if an economical method for preparing fine particles can be established.

\footnotetext{
* Corresponding author. Tel./fax: +886223620832.

E-mail address: cytai@ntu.edu.tw (C.Y. Tai).
}

There are two major methods for preparing $\mathrm{BaCO}_{3}$, based on the type of reactant employed, i.e., carbonation by means of $\mathrm{CO}_{2}$ feeding and precipitation by mixing two liquid feeds. For carbonation, $\mathrm{BaCO}_{3}$ is prepared by introducing $\mathrm{CO}_{2}$ into a solution containing barium sulfide or barium hydroxide. It can also be prepared through reactive precipitation caused by a reaction between two liquid feeds of barium and carbonate aqueous solutions. Yagi et al. (1988) utilized a carbonation route for preparing $\mathrm{BaCO}_{3}$ in a batch precipitator under a controlled $\mathrm{pH}$. The obtained $\mathrm{BaCO}_{3}$ appeared in the form of flocs with sizes ranging from 10 to $30 \mu \mathrm{m}$. Kubota et al. (1990) also applied a carbonation route for preparing $\mathrm{BaCO}_{3}$ in a batch precipitator, where the local supersaturation was controlled by using a single-tube or a double-tube mode to introduce $\mathrm{CO}_{2}$. Pillar-like $\mathrm{BaCO}_{3}$, ranging from 3 to $7 \mu \mathrm{m}$ in size, was obtained by applying the double-tube mode. On the other hand, needlelike $\mathrm{BaCO}_{3}$, ranging from 3 to $5 \mu \mathrm{m}$ in size, was obtained when a single-tube was chosen. Using a double-jet feeding method, Chen et al. (2001) prepared $\mathrm{BaCO}_{3}$ in a batch precipitator, in which the environment was kept at a constant $\mathrm{pH}$. Particles larger than $10 \mu \mathrm{m}$ were obtained with different morphologies, including floc, candy-like, olivary and needle-like shapes. Recently, with the aid of urease, urea was decomposed to release $\mathrm{CO}_{2}$ in an aqueous solution of barium salts, so as to form 
uniform and sphere-like $\mathrm{BaCO}_{3}$ particles with a size of $3 \mu \mathrm{m}$ via fast precipitation (Sondi and Matijevic, 2003). In the studies mentioned above, only micron particles or larger ones were obtained in a stirred batch precipitator.

The higee technique, proposed by Ramshaw (1995), is an example of process intensification and is designed to minimize the equipment scale, to save space, resources and energy, and thus to make the chemical industry cleaner and safer. During the past two decades, higee systems have been extensively applied due to the great enhancement in the mass-transfer rate in many unit operations, such as distillation, absorption, stripping, extraction, and adsorption, and it has recently been adopted in the field of precipitation. A report by Chen et al. (2000) indicated that submicron and even nano particles, including $\mathrm{CaCO}_{3}$, $\mathrm{SrCO}_{3}$, and $\mathrm{Al}(\mathrm{OH})_{3}$, were successfully produced by a highgravity rotating packed bed (HGRPB) reactor without adding surfactants to the reacting solution. In another report, Cafiero et al. (2002) prepared $\mathrm{BaSO}_{4}$ particles with a mean size of $0.7 \mu \mathrm{m}$ by using a higee-creating spinning disk reactor (SDR), in which uniform distribution of supersaturation due to high mixing efficiency was essential for obtaining particles with a narrow size distribution. The flow pattern in higee equipment is highly turbulent and complex while mixing proceeds, and the turbulent eddy size is usually used to define the mixing efficiency. As the turbulent eddy size approaches to molecular level, a state of highly efficient mixing, called micromixing, is achieved. Chen et al. (2006) applied a two-parallel-competingreaction mode to study the mixing efficiency, using the segregation index $\left(X_{S}\right)$ as the criterion. When $X_{S}$ equals zero, perfect mixing is achieved. On the other hand, poor mixing results when $X_{s}$ approaches 1 . The segregation index of a SDR is that which most closely approaches zero as compared with those of previously studied mixers, including a rotating packed bed reactor (RPBR), a static mixer, a Couette flow reactor, and a constant stirred tank reactor (Chen et al., 2006).

In this research, a less studied system, barium carbonate, was chosen as a model compound to investigate the possibility of producing fine particles using a SDR. The precipitation of $\mathrm{BaCO}_{3}$ was carried out via a carbonation route and a oncethrough mode, and the effects of the operation variables on the particle size of $\mathrm{BaCO}_{3}$ were investigated, including the $\mathrm{CO}_{2}$ flow rate, the feed rate of $\mathrm{Ba}(\mathrm{OH})_{2}$ slurry, the rotation speed, and the solid-content of feed slurry. The particle size distribution was measured using a laser-light analyzer after a suitable dispersion method was applied to obtain reproducible particle size data. The collected particles were characterized using a scanning electronic microscope to determine the particle size and shape, and an X-ray diffractometer was used to study the crystal morphology.

\section{Experimental}

\subsection{Higee equipment}

A schematic diagram of the Higee system used in this study is shown in Fig. 1(a). It consisted of a $\mathrm{CO}_{2}$ feeding system, a slurry feeding system, a higee reactor, and a slurry collection

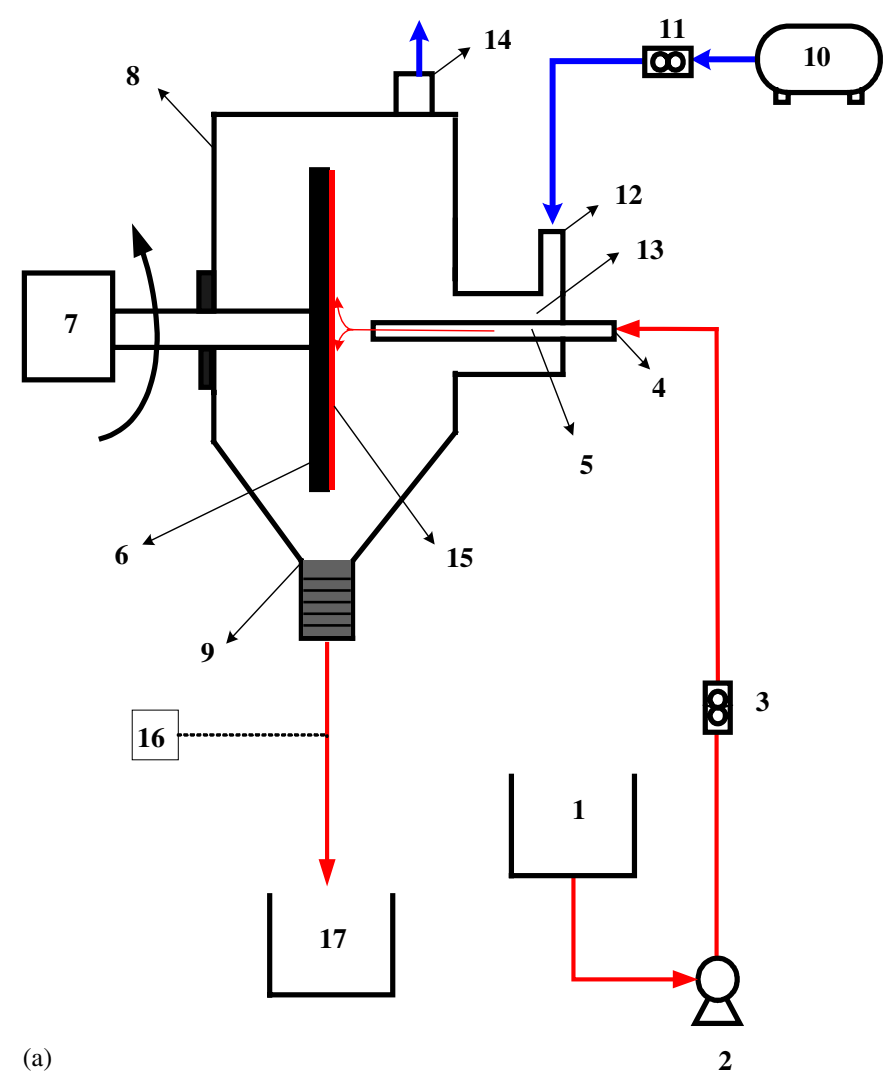

(b)

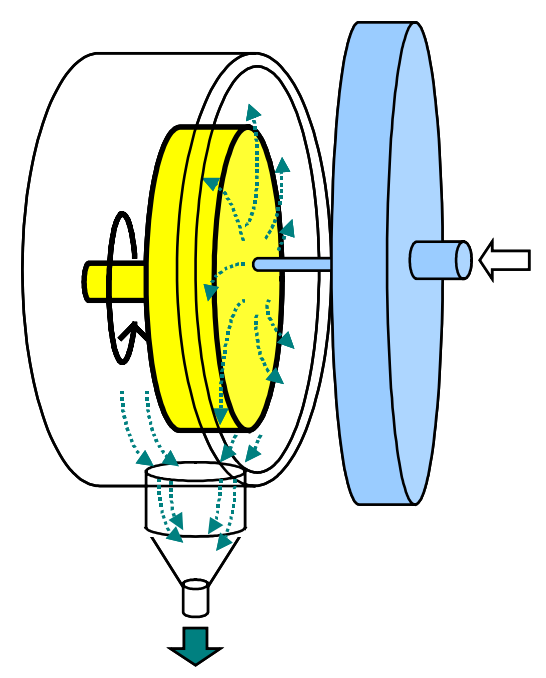

Fig. 1. (a) A schematic diagram of a higee system for producing $\mathrm{BaCO}_{3}$ particles via a carbonation route; (b) a spinning disk reactor.

vessel. The main part was a stainless-steel disk (6), $12 \mathrm{~cm}$ in diameter, driven by a variable-speed motor (7). The spinning disk was enclosed in a cylindrical acrylic-chamber (8) that was $15 \mathrm{~cm}$ in diameter and $6 \mathrm{~cm}$ deep. A funnel-shaped outlet (9) was located at the bottom of the chamber and used to guide the slurry into a collection vessel (17). The stem of the funnelshaped outlet was used as a liquid seal to prevent $\mathrm{CO}_{2}$ from escaping from the bottom of reactor. The entire setup is called a SDR and is shown in Fig. 1(b). In addition, slurry reactant was introduced into the reactor through a feeding distributor (5). 


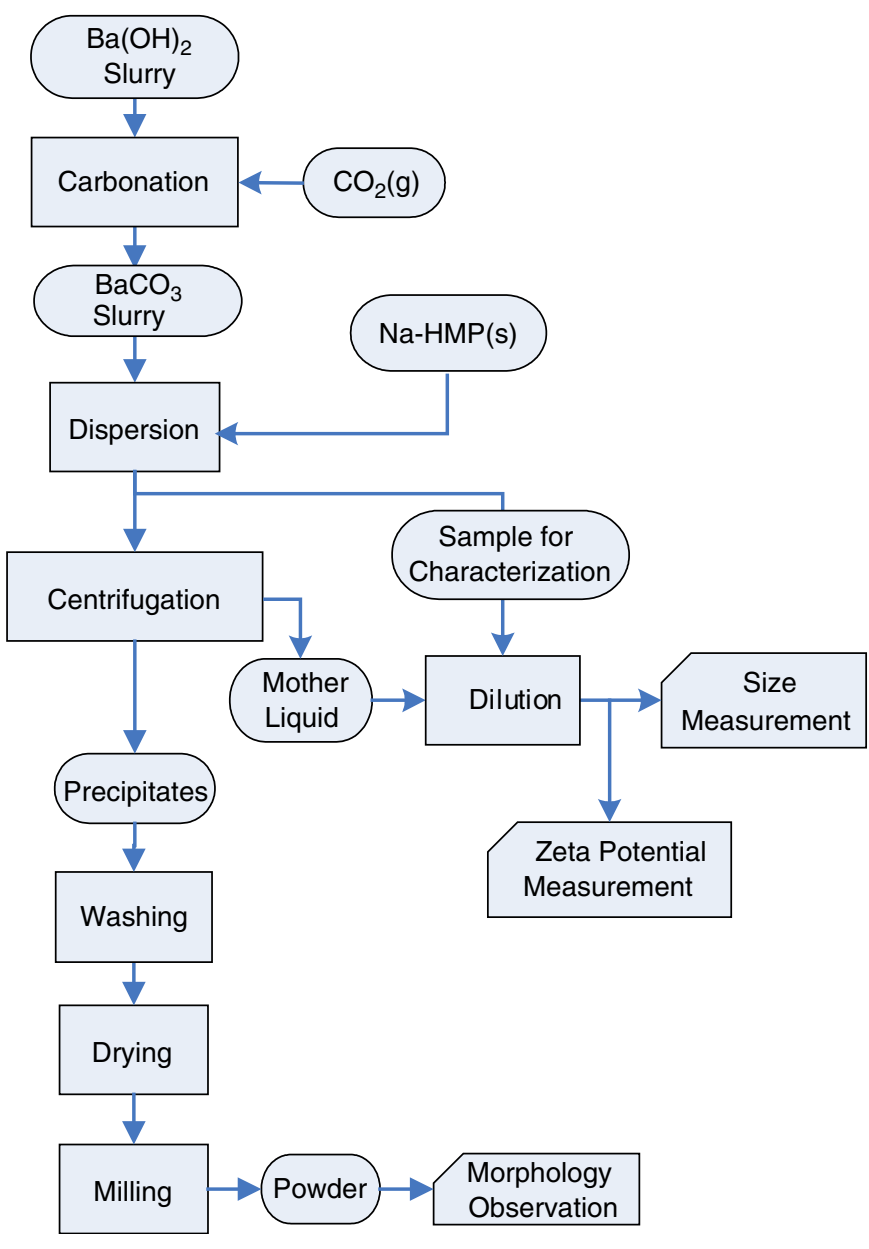

Fig. 2. A flow chart of the experimental procedure for producing and characterizing $\mathrm{BaCO}_{3}$ particles.

\subsection{Experimental procedure}

A flow chart of the experimental procedures is presented in Fig. 2. The material fed into the reactor for carbonation under ambient conditions was a slurry composed of barium hydroxide with a desired solid-content, ranging from 10 to $55 \mathrm{~g} / \mathrm{L} \mathrm{H}_{2} \mathrm{O}$. The slurry was first charged into a storage tank (1) and then pumped into the chamber (8) after the motor (7) had been switched on to drive the spinning disk with a rotation speed ranging from 600 to $2100 \mathrm{rpm}$. The flow rate of slurry feeding was set between 250 and $850 \mathrm{ml} / \mathrm{min}$, and measured with a rotameter (3). The other feeding stream of $\mathrm{CO}_{2}$ gas from a cylinder (10) was charged into the chamber with a flow rate ranging from 1.5 to $6 \mathrm{NL} / \mathrm{min}$.

For the SDR, the feeding slurry was introduced through a distributor (5) onto the center of the disk (6). The distributor was a straight tube with a $1 \mathrm{~mm}$-diameter hole. Then, the slurry was accelerated on the disk surface to form a thin film (15), where the dissolved barium carbonate reacted with the absorbed $\mathrm{CO}_{2}$ to produce $\mathrm{BaCO}_{3}$ particles. The slurry containing $\mathrm{BaCO}_{3}$ then left the periphery of the disk to hit the inner wall of the chamber (8), and flowed down along the wall then past the funnel-like outlet (9), where some slurry remained and formed a liquid seal, preventing $\mathrm{CO}_{2}$ from escaping. The remaining slurry went into a collection vessel (17). An on-line $\mathrm{pH}$ probe (16) was used to measure the $\mathrm{pH}$ value of the slurry at the outlet of the reactor to ensure complete carbonation, based on a $\mathrm{pH}$ value reading that was below 7 .

After the carbonation step was completed, a certain amount of sodium hexametaphosphate (Na-HMP), ranging from 0 to $35.2 \mathrm{~g} / \mathrm{kg}$ slurry, was added to the slurry to disperse $\mathrm{BaCO}_{3}$ particles. The dispersed sample was further diluted for size and zeta potential measurements. A static light scattering analyzer (Coulter, LS230) was used to determine the particle size distribution, and a dynamic light scattering analyzer (Malvern, Mastersizer) was used to measure the zeta potential. Meanwhile, a portion of the slurry was centrifuged under $15,000 \mathrm{~g}$ for $5 \mathrm{~min}$, washed with deionized water three times and with a $99 \%$ ethanol solution twice, then dried overnight at $80^{\circ} \mathrm{C}$, and finally bowl-milled for $5 \mathrm{~min}$ to obtain dried precipitates for morphology determination, using a scanning electronic microscope (JEOL, J-5600) and an X-ray diffractometer (Mac Science, MXP-3TXJ-7266).

\section{Results and discussion}

\subsection{Dispersion of $\mathrm{BaCO}_{3}$ particles}

Before performing a systematic study of the operation variables of a Higee system for producing particles, one needs to establish a reliable size-measuring method by means of which reproducible PSD data can be collected in order to identify the factors that affect the particle size. To obtain reproducible size data using a laser-light scattering particle size analyzer, barium carbonate particles should be well dispersed in an aqueous solution. Na-HMP is the most commonly used dispersant for sparingly soluble carbonate salts because of its high dispersing ability, low price and easy availability. In our experiment, three slurry samples of different solid-contents, i.e., $0.63,1.66$, and $3.23 \mathrm{wt} \%$, were prepared under the following conditions with a $\mathrm{CO}_{2}$ flow rate of $5 \mathrm{NL} / \mathrm{min}$, a slurry feed rate of $250 \mathrm{ml} / \mathrm{min}$, a rotation speed of $2100 \mathrm{rpm}$, and three different solid-contents of feeding slurry, i.e., $10,27.6$ and $55 \mathrm{~g} \mathrm{Ba}(\mathrm{OH})_{2} / \mathrm{L} \mathrm{H}_{2} \mathrm{O}$ for the three corresponding samples, respectively. The changes in the $\mathrm{pH}$ value, weight loss and zeta-potential of the three samples were recorded for different dosages of Na-HMP, ranging from 0 to $35.2 \mathrm{~g} / \mathrm{kg}$ slurry.

The changes in the $\mathrm{pH}$ value and zeta potential of the three samples for different dosages of Na-HMP are shown in Fig. 3(a)-(c), respectively. Apparently, the $\mathrm{pH}$ value increased with an increase in the dosage of Na-HMP, and then the $\mathrm{pH}$ value leveled off at a dosage of approximately $5 \mathrm{~g} / \mathrm{kg}$ slurry. As for the zeta potential, a similar trend was observed; i.e., the zeta potential changed drastically under a dosage of less than $5 \mathrm{~g}$ Na-HMP/kg slurry. In addition, the weight loss of the three slurry samples increased with an increase in the dosage of Na-HMP, as shown in Fig. 4(a)-(c). The procedure for measuring the weight loss of slurry solution is described below. After carbonation we collected a sample of $\mathrm{BaCO}_{3}$ slurry, which was weighted as $W$. Then, a certain amount of Na-HMP, 

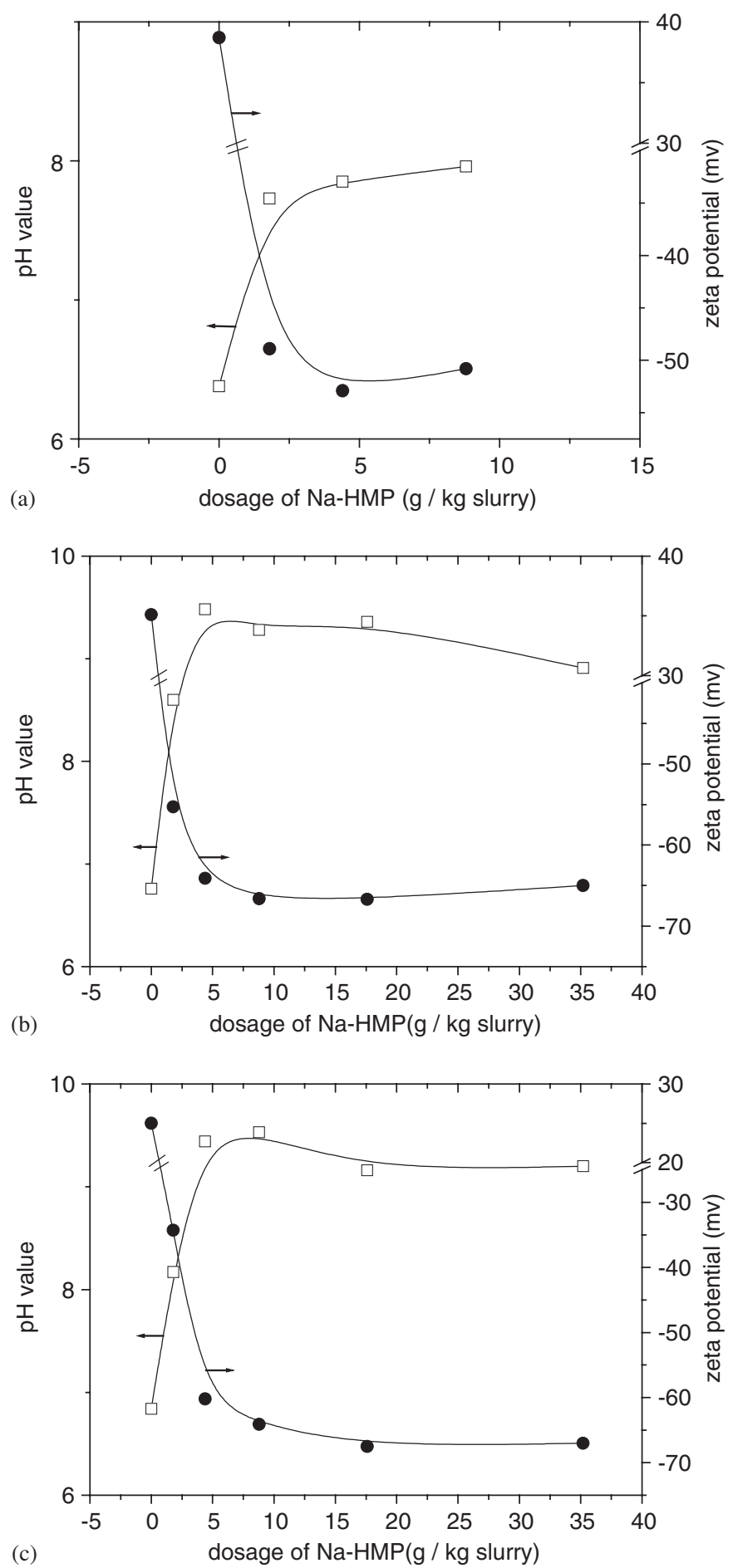

Fig. 3. Zeta potential and $\mathrm{pH}$ value for three slurry samples with different solid-contents after adding different amounts of Na-HMP: (a) $0.63 \mathrm{wt} \%$; (b) $1.66 \mathrm{wt} \%$; and (c) $3.23 \mathrm{wt} \%$.

ranging from 0 to $35.2 \mathrm{~g} / \mathrm{kg}$ slurry was added into the slurry for dispersing the particles. Finally, the solid precipitate was separated from slurry by centrifugation, followed by washing, drying and was weighted as $W_{s}$. The solid-content of slurry was calculated as $\left(W_{s} / W\right) \times 100 \%$, which was denoted by $\Phi$ and $\Phi_{0}$ for the slurry with and without adding Na-HMP
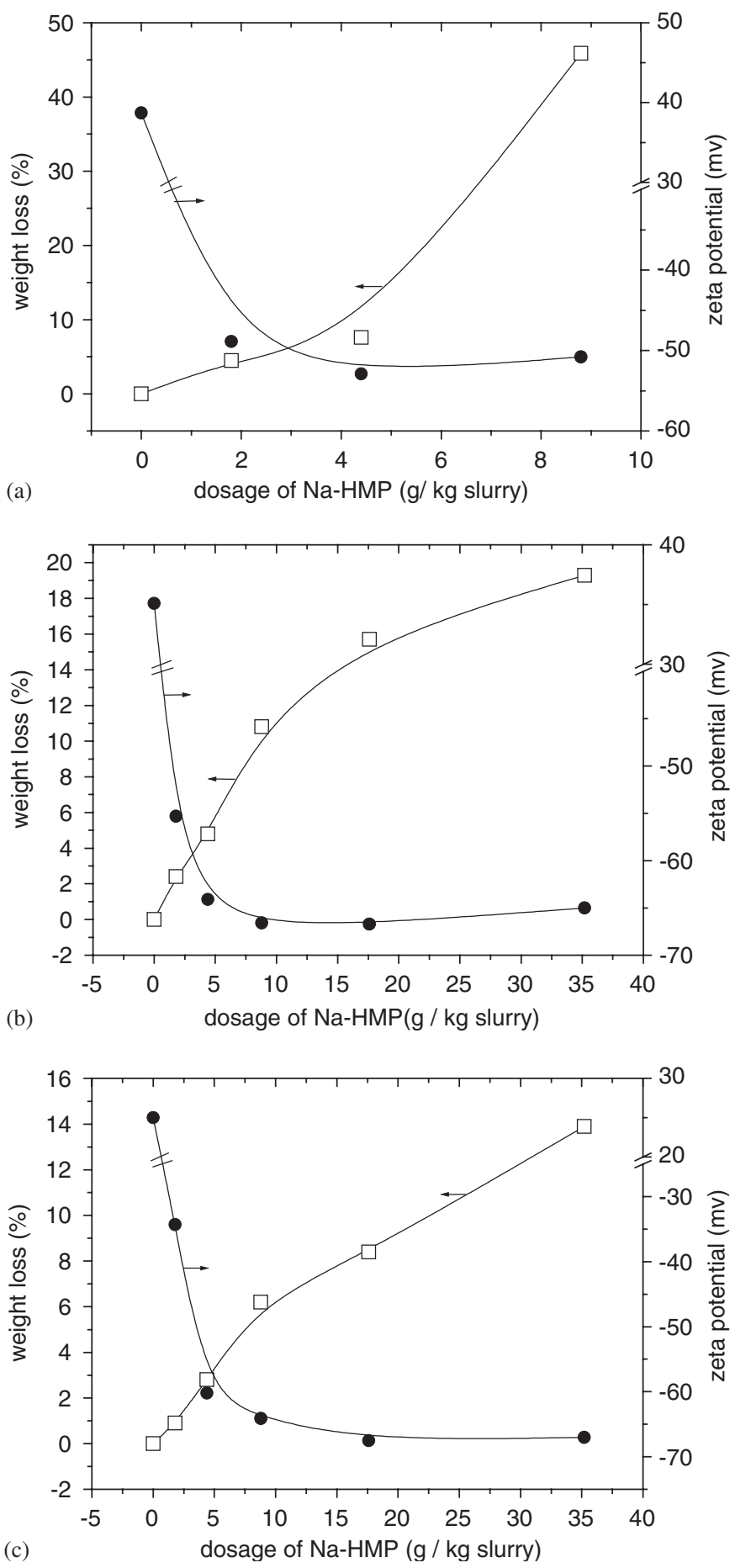

Fig. 4. Weight loss and zeta potential of three slurry samples with different solid-contents after adding different amounts of Na-HMP: (a) $0.63 \mathrm{wt} \%$; (b) $1.66 \mathrm{wt} \%$; and (c) $3.23 \mathrm{wt} \%$.

separately. The weight loss was defined as $\left[\left(\Phi_{0}-\Phi\right) / \Phi_{0}\right] \times$ $100 \%$. The weight loss of the slurry implies a certain degree of dissolution of $\mathrm{BaCO}_{3}$ particles after Na-HMP was added. For instance, the particles of the $0.63 \mathrm{wt} \%$ sample were almost dissolved when the Na-HMP dosage was $17.6 \mathrm{~g} / \mathrm{kg}$ slurry, where the zeta potential could not be measured. Thus, the data points at the two Na-HMP dosages, i.e., 17.6 and $35.2 \mathrm{~g} / \mathrm{kg}$ slurry, were not presented in Figs. 3(a) and 4(a). 

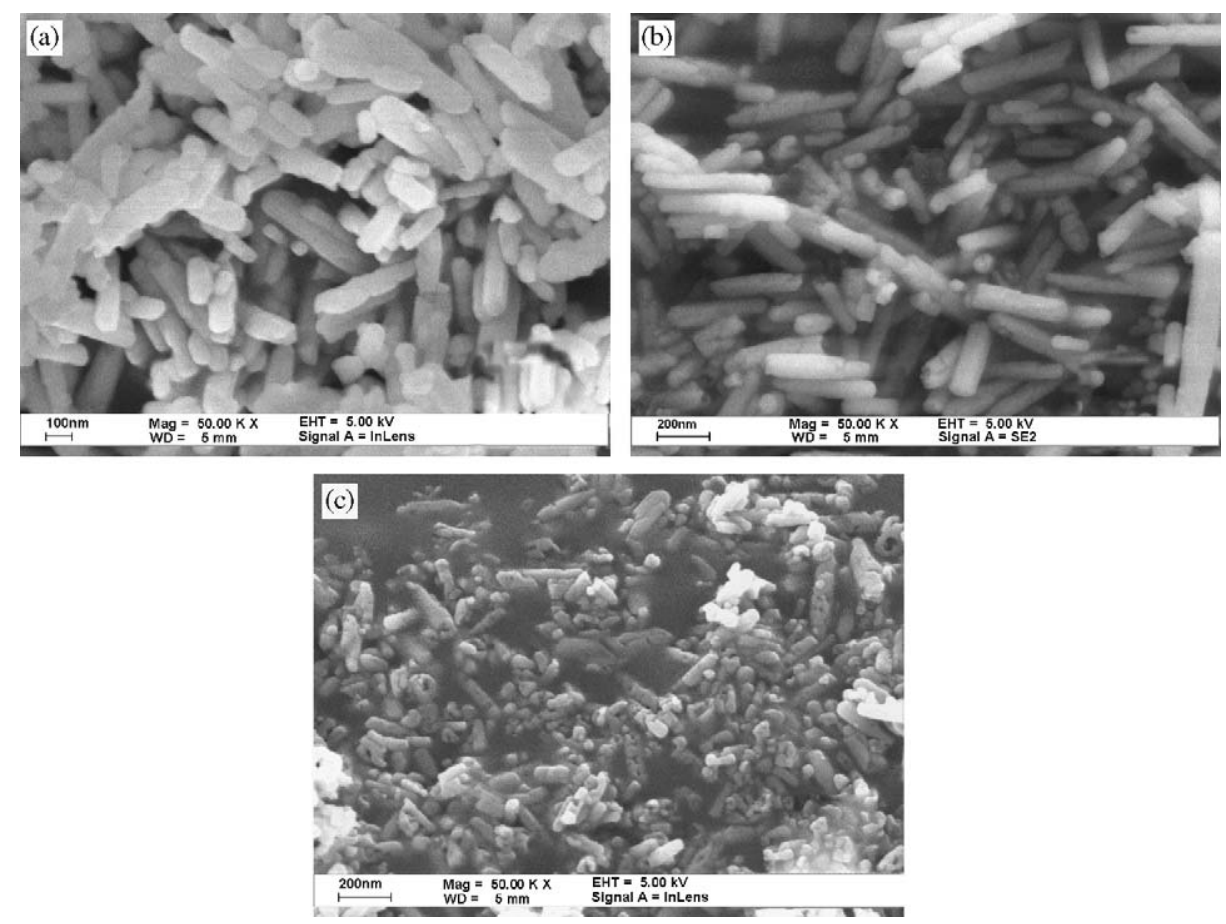

Fig. 5. A comparison of the $\mathrm{BaCO}_{3}$ morphology under different dosages of Na-HMP: (a) without adding Na-HMP; (b) $4.4 \mathrm{~g}$ Na-HMP/kg slurry; (c) $35.2 \mathrm{~g}$ Na-HMP / kg slurry.

The action of phosphate as a deflocculant was reviewed in detail by Lyons (1961). The phosphate Na-HMP as a polyelectrolyte ionizes to a highly negative-charged ion when it dissolves in water. On the highly negative-charged ion, there exist many electron-abundant oxygen atoms, every pair of which act as a ligand and tend to chelate with an alkaline-earth metallic ion (Lyons, 1961; Dwyer and Mellor, 1964). In addition, ionized Na-HMP can be adsorbed to the surface of colloids by means of metallic chelation and/or an electrostatic force, providing sufficient electrostatic repulsion to disperse particles (Griffith et al., 1973). The following mechanism is proposed to explain the phenomena observed in Figs. 3 and 4. When the dissolved Na-HMP ionized in water, every pair of electron-abundant oxygen atoms on it acted as a ligand to chelate electron-deficient barium on the surface of $\mathrm{BaCO}_{3}$ particles, resulting in the breaking of the original ionic bonds between the barium and carbonate on the surface. Due to the breakage of ionic bonds, particles started to dissolve to a certain degree, depending on the amount of added Na-HMP. At the same time, ionized NaHMP was attracted to a particle's surface by means of metallic chelation or some other force such that the particle's surface was loaded with highly negative charges. On the other hand, some carbonate ions were released from a particle's surface upon the breakage of ionic bonds, and the "free" carbonate ions, acting as weak alkali, neutralized the protons in the solution. This could explain the increase in the $\mathrm{pH}$ value of slurry after Na-HMP was added.

Generally speaking, a zeta-potential of $-40 \mathrm{mv}$ was sufficient to disperse submicron particles. As shown in Fig. 3(a)-(c), the zeta-potential of $\mathrm{BaCO}_{3}$ particles could easily reach $-50 \mathrm{mv}$ after a certain amount of Na-HMP was added, i.e., approximately $2 \mathrm{~g} / \mathrm{kg}$ slurry. As a result, Na-HMP produced highly negative charges on a particle's surface, resulting in stabilization of the slurry. However, a higher dosage of Na-HMP caused the dissolution of $\mathrm{BaCO}_{3}$ particles and, subsequently, changed the PSD and morphology of the originally prepared products. As shown in Fig. 5(a)-(c), when the weight loss of the $1.66 \mathrm{wt} \%$ sample with a dosage of $4.4 \mathrm{~g} \mathrm{Na}-$ $\mathrm{HMP} / \mathrm{kg}$ slurry was approximately $5 \%$, the size and morphology of the $\mathrm{BaCO}_{3}$ particles changed very little as compared with that when no Na-HMP was added. However, the size and aspect ratio apparently became smaller when the weight loss was $19 \%$ with a dosage of $35.2 \mathrm{~g} \mathrm{Na}-\mathrm{HMP} / \mathrm{kg}$ slurry. To achieve a compromise between excessive dissolution and a sufficient zeta potential, a dosage of Na-HMP that yielded a weight loss of slurry of less than 5\% and a zeta-potential of less than $-50 \mathrm{mv}$ was chosen. From Fig. 4(a)-(c), the appropriate Na-HMP dosages for solid-contents of $0.63,1.66$, and $3.23 \mathrm{wt} \%$ were $1.8,4.4$ and $4.4 \mathrm{~g}$, respectively.

As far as the morphology is concerned, only one type of $\mathrm{X}$-ray diffraction pattern was obtained in this study, as shown in Fig. 6, which matched the one numbered 41-0373 in JCPDS for orthorhombic $\mathrm{BaCO}_{3}$.

\subsection{Effect of the $\mathrm{CO}_{2}$ flow rate on the PSD of $\mathrm{BaCO}_{3}$}

The factors that affected the PSD of $\mathrm{BaCO}_{3}$, including the $\mathrm{CO}_{2}$ flow rate, the feed rate of $\mathrm{Ba}(\mathrm{OH})_{2}$ slurry, the rotation speed, and the solid-content of the feed slurry, were investigated using the SDR. The $\mathrm{CO}_{2}$ flow rate had little impact on 


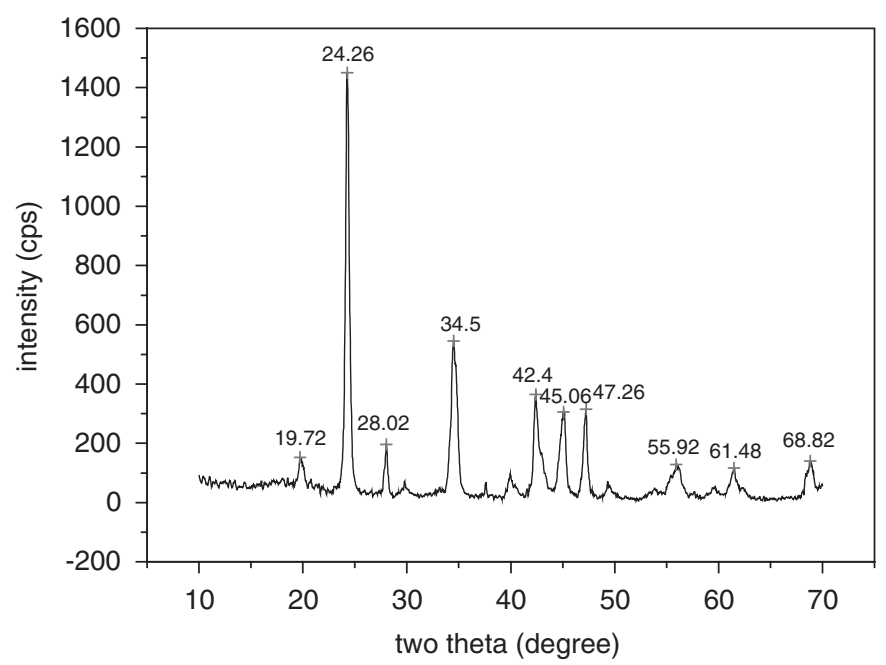

Fig. 6. X-ray diffraction pattern of $\mathrm{BaCO}_{3}$ prepared in this study.

Table 1

Effects of rotation speed and slurry feed rate on the PSD of $\mathrm{BaCO}_{3}$

\begin{tabular}{llll}
\hline $\begin{array}{l}\text { Rotation speed } \\
(\mathrm{rpm})\end{array}$ & $\begin{array}{l}\text { Mean size } \\
(\mathrm{nm})\end{array}$ & $\begin{array}{l}\text { Standard deviation } \\
(\mathrm{nm})\end{array}$ & $\begin{array}{l}\text { Standard deviation/ } \\
\text { mean size }\end{array}$ \\
\hline \multicolumn{2}{l}{ Feed rate $=250 \mathrm{ml} / \mathrm{min}$} & & \\
600 & 366 & 463 & 1.27 \\
1200 & 366 & 472 & 1.29 \\
1800 & 348 & 466 & 1.34 \\
2100 & 339 & 420 & 1.24 \\
& & & \\
Feed rate $=850 \mathrm{ml} / \mathrm{min}$ & & - \\
$600^{\mathrm{a}}$ & - & - & 1.14 \\
1200 & 700 & 797 & 1.19 \\
1800 & 641 & 760 & 1.45 \\
2100 & 490 & 710 & \\
\hline
\end{tabular}

${ }^{\mathrm{a}}$ Carbonation was not completed.

the particle size. For example, when the flow rate was varied from 1.5 to $6 \mathrm{~L} / \mathrm{min}$, the volume mean size changed from 345 to $339 \mathrm{~nm}$. The effects of the other operating variables are presented below.

\subsection{Effects of the rotation speed and feed rate on the PSD of $\mathrm{BaCO}_{3}$}

Table 1 shows the effects of the rotation speed, which was varied from 600 to $2100 \mathrm{rpm}$, on the PSD of $\mathrm{BaCO}_{3}$ particles under two different liquid feed rates of slurry, i.e., at 250 and $850 \mathrm{ml} / \mathrm{min}$, while the solid-content of the feed slurry and the $\mathrm{CO}_{2}$ flow rate were fixed. Data for the operating conditions at $850 \mathrm{ml} / \mathrm{min}$ and $600 \mathrm{rpm}$ are lacking, because the carbonation reaction was not completed under the operating conditions. Also listed in the table is the ratio between the standard deviation and mean size, which indicates the uniformity of the size distribution. A general trend is observed for both feed rates: the particles size decreased with an increase in the rotation speed. However, the effect of the rotation speed was very significant in

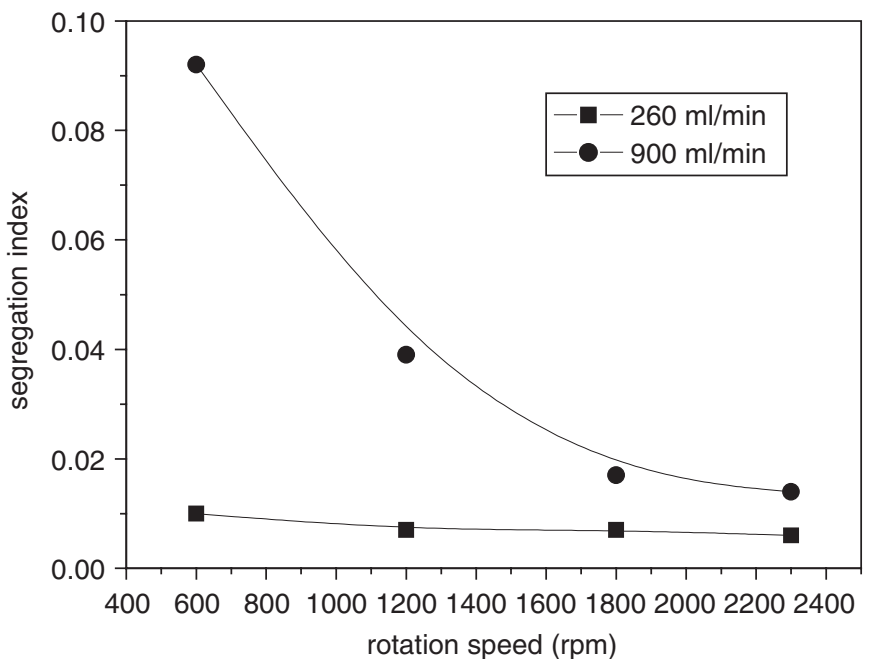

Fig. 7. A comparison of the segregation index for two feed rates of 260 and $900 \mathrm{ml} / \mathrm{min}$ under different rotation speeds (Chen et al., 2006).

the case of a high flow rate; a change from 366 to $339 \mathrm{~nm}$, i.e., $7 \%$ decrease in the mean size, was obtained under a flow rate of $250 \mathrm{ml} / \mathrm{min}$ as compared with $30 \%$ decrease, i.e., a change from 700 to $490 \mathrm{~nm}$, under a flow rate of $850 \mathrm{ml} / \mathrm{min}$. As far as the effect of the feed rate is concerned, a low feed rate produced a small particle size. At a low rotation speed of $1200 \mathrm{rpm}$, the mean size decreased from 700 to $366 \mathrm{~nm}$ as the feed rate changed from 850 to $250 \mathrm{ml} / \mathrm{min}$. However, the decrease was much smaller at a higher rotation speed of $2100 \mathrm{rpm}$. For example, the mean size decrease from 490 to $339 \mathrm{~nm}$. These effects can be explained by the mixing efficiency and $\mathrm{CO}_{2}$ absorption capacity of $\mathrm{Ba}(\mathrm{OH})_{2}$ slurry.

Cafiero et al. (2002) stated that a thin liquid film can be formed by introducing a liquid onto the center of a spinning disk, as in the process of spin coating. Inside the thin film, there exists a high level of turbulence. Increasing the rotation speed will improve the mixing efficiency, because a smaller turbulent eddy size will produce more intense and efficient mixing. The results reported by Chen et al. (2006) support the view of Cafiero et al. (2002), as shown in Fig. 7, in which the segregation index is plotted against the rotation speed. The segregation index, varied from 0 to 1 , represents for the degree of mixing; 0 means perfect mixing, and 1 indicates poor mixing. Detail discussion and calculation of the segregation index can be found in the literature (Fournier et al., 1996). In Fig. 7, a general trend is observed, where the segregation index decreases with increasing rotation speed for the two different feed rates. The segregation indexes for a feed rate of $260 \mathrm{ml} / \mathrm{min}$ are smaller than 0.01 , almost independent of the rotation speed, and lower than those for a feed rate of $900 \mathrm{ml} / \mathrm{min}$. It is understood that a thicker film will form on the rotating disk when the feed rate is higher. Thus, mixing will be less effective under a high feed rate. Based on the surface renewal concept, efficient mixing at a low feed rate will provide more fresh interfaces for $\mathrm{CO}_{2}$ absorption, thus increasing the transfer rate. A low feed rate and high $\mathrm{CO}_{2}$ transfer rate will result in high supersaturation, 
Table 2

Effect of solid-content of feed $\mathrm{Ba}(\mathrm{OH})_{2}$ slurry on the PSD of $\mathrm{BaCO}_{3}$

\begin{tabular}{llll}
\hline $\begin{array}{l}\text { Solid-content of } \\
\mathrm{Ba}(\mathrm{OH})_{2} \text { slurry } \\
(\mathrm{g} / \mathrm{L} \text { water })\end{array}$ & $\begin{array}{l}\text { Mean size } \\
(\mathrm{nm})\end{array}$ & $\begin{array}{l}\text { Standard } \\
\text { deviation } \\
(\mathrm{nm})\end{array}$ & $\begin{array}{l}\text { Standard } \\
\text { deviation/ } \\
\text { mean size }\end{array}$ \\
\hline 10.0 & 731 & 769 & 1.05 \\
27.6 & 339 & 420 & 1.24 \\
55.0 & 915 & 946 & 1.03 \\
\hline
\end{tabular}

which will result in a high nucleation rate and, thus, smaller particles. Once the particles formed, they left the SDR immediately and the solution concentration dropped to saturation. The particles got no chance to grow. Therefore, the effect of growth rate on particle size was not considered here. This is shown in Table 1, where the mean particle size at the low feed rate of $250 \mathrm{ml} / \mathrm{min}$ and the highest rotation speed of $2100 \mathrm{rpm}$ is the smallest among the experimental data obtained under various operating conditions. On the other hand, although a difference in the mixing efficiency achieved under the two feed rates is observed in Fig. 7, the mixing efficiency at the high feed rate was high enough to give a uniformity of PSD represented by the ratio of the standard deviation to the mean size listed in Table 1, which is as good as that at the low feed rate.

\subsection{Effect of the solid-content of the feed slurry on the PSD of $\mathrm{BaCO}_{3}$}

We also studied the effect of the solid-content of the feed slurry on the PSD of products by varying the solid-content of the feed slurry from 10 to $55 \mathrm{~g} \mathrm{Ba}(\mathrm{OH})_{2} / \mathrm{L} \mathrm{H}_{2} \mathrm{O}$. The results shown in Table 2 indicate that the minimum mean size was obtained with a solid-content of $27.6 \mathrm{~g} \mathrm{Ba}(\mathrm{OH})_{2} / \mathrm{L} \mathrm{H}_{2} \mathrm{O}$. This minimum size was also obtained in an experiment using a RPBR (Tai and Tai, 2005), which is another type of higee equipment. Referring to Table 1, which indicates that carbonation was not completed under some specific conditions, a possible $\mathrm{pH}$ profile along the radial direction of a spinning disk is proposed and shown in Fig. 8 to explain the minimum mean size. For the once-through carbonation process carried out in our study, the $\mathrm{pH}$ value dropped to a value between 6 and 7 at some point on the disk where the carbonation was completed. Before this point was reached, a high $\mathrm{pH}$ value plateau was maintained for a certain distance from the center of the disk, indicating a saturated $\mathrm{Ba}(\mathrm{OH})_{2}$ solution, in which the amount of reacted $\mathrm{Ba}(\mathrm{OH})_{2}$ was replenished by dissolving $\mathrm{Ba}(\mathrm{OH})_{2}$ solids. Before the dissolution of $\mathrm{Ba}(\mathrm{OH})_{2}$ solids was completed, the $\mathrm{pH}$ value entered a transition state, and the solution finally became acidic before leaving the disk.

With the increase of the solid-content of the $\mathrm{Ba}(\mathrm{OH})_{2}$ feed slurry, more solids were provided to replace the $\mathrm{Ba}(\mathrm{OH})_{2}$ lost in the liquid phase as carbonation proceeded. Therefore, a longer high $\mathrm{pH}$ value plateau on a disk was maintained, resulting in high supersaturation for a longer distance and finer particles. However, the transition state of the $\mathrm{pH}$ value also lasted for a longer distance under a high solid-content of $\mathrm{Ba}(\mathrm{OH})_{2}$ slurry

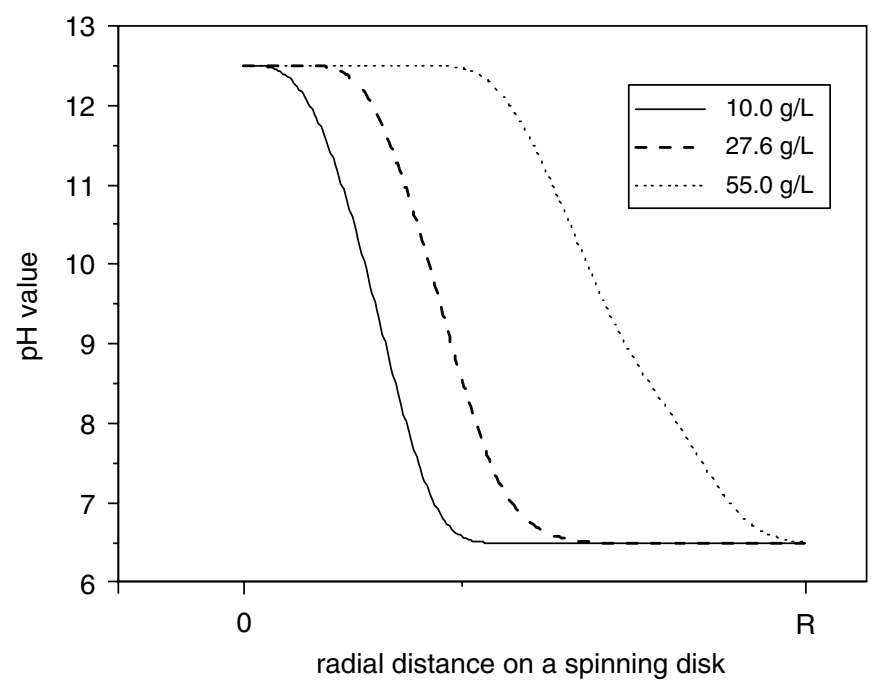

Fig. 8. A possible $\mathrm{pH}$ profile along the radial direction on a spinning disk for the three solid-contents of feed $\mathrm{Ba}(\mathrm{OH})_{2}$ slurry.

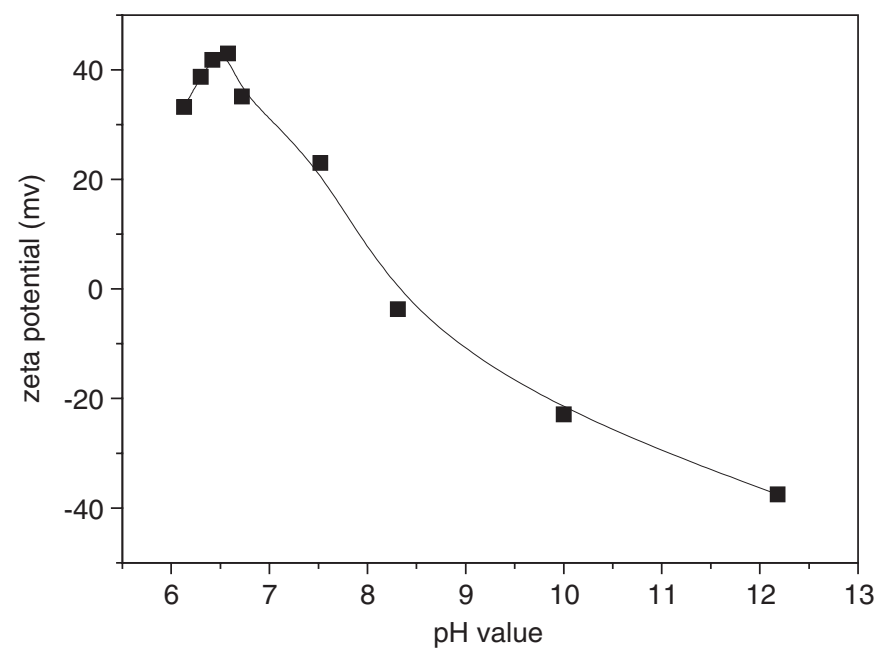

Fig. 9. Zeta potential of $\mathrm{BaCO}_{3}$ as a function of $\mathrm{pH}$.

because more surface area of $\mathrm{Ba}(\mathrm{OH})_{2}$ particles was available for dissolving $\mathrm{Ba}(\mathrm{OH})_{2}$. This means that the $\mathrm{pH}$ value in the high solid-content run could not drop suddenly to below 7 as in the lower solid-content runs, in which the $\mathrm{Ba}(\mathrm{OH})_{2}$ solids were fewer in number and disappeared quickly once the transition state appeared. The zeta potential values measured at different $\mathrm{pH}$ values between 6.1 and 12.2 are shown in Fig. 9. The zeta potential was below $30 \mathrm{mv}$ of absolute value, which is lower than the value of $40 \mathrm{mv}$ for dispersing submicron particles, in the $\mathrm{pH}$ value range between 7 and 12 . The $\mathrm{pH}$ range between 7 and 12 simulated the $\mathrm{pH}$ transition of slurry on the spinning disk. Thus, when the solid-content was increased from 10 to $27.6 \mathrm{~g} / \mathrm{L}$, a longer high $\mathrm{pH}$ value plateau, and thus a longer period of high supersaturation was maintained on the disk to 
produce smaller particles. However, with a further increase of the solid-content to $55 \mathrm{~g} / \mathrm{L}$, agglomeration became much more significant during the longer $\mathrm{pH}$ transition state, because of the low absolute value of the zeta potential. Thus, the mean size of the $\mathrm{BaCO}_{3}$ particles increased when the solid-content was $55 \mathrm{~g}$ $\mathrm{Ba}(\mathrm{OH})_{2} / \mathrm{L} \mathrm{H} \mathrm{H}_{2} \mathrm{O}$.

\section{Conclusion}

Rod-shaped submicron $\mathrm{BaCO}_{3}$ particles were successfully prepared by using a spinning disk reactor, a type of higee equipment, due to the achieved mixing efficiency and high transfer rate of $\mathrm{CO}_{2}$ generated in the reactor.

In order to study the factors that affected the particle size, a reliable size-measuring method was established. The produced particles were dispersed in water using sodium hexametaphosphate as a dispersant; thus, reliable particle size distribution data could be obtained, using a static light-scattering size analyzer. The operating variables were then systematically studied, including the $\mathrm{CO}_{2}$ flow rate, the feed rate of $\mathrm{Ba}(\mathrm{OH})_{2}$ slurry, the rotation speed, and the solid-content of the feed slurry. Among them, the effects of the feed rate and the solid-content of $\mathrm{Ba}(\mathrm{OH})_{2}$ slurry were most significant as far as the particle size was concerned. A low feed rate led to a small particle size, and a minimum size was obtained when the solid-content ranged from 10.0 to $55.0 \mathrm{~g} \mathrm{Ba}(\mathrm{OH})_{2} / \mathrm{L} \mathrm{H}_{2} \mathrm{O}$. At a low feed rate of $250 \mathrm{ml} / \mathrm{min}$, the rotation speed had no effect on the particle size; however, the effect of rotation speed was significant at a high flow rate of $850 \mathrm{ml} / \mathrm{min}$.

\section{Acknowledgment}

The authors gratefully acknowledge the financial support provided by the Ministry of Economic Affairs, Taiwan, the Republic of China.

\section{References}

Cafiero, L.M., Baffi, G., Chianese, A., Jachuck, R.J.J., 2002. Process intensification: precipitation of barium sulfate using a spinning disk reactor. Industrial Engineering \& Chemistry Research 41, 5240-5246.

Chen, J.F., Wang, Y.H., Guo, F., Wang, X.M., Zheng, C., 2000. Synthesis of nanoparticles with novel technology: high-gravity reactive precipitation. Industrial Engineering \& Chemistry Research 39, 948-954.

Chen, P.C., Cheng, G.Y., Kou, M.H., Shia, P.Y., Chung, P.O., 2001. Nucleation and morphology of barium carbonate crystals in a semi-batch crystallizer. Journal of Crystal Growth 226, 458-472.

Chen, Y.S., Tai, C.Y., Chang, M.H., Liu, H.S., 2006. Characteristics of micromixing in a rotating packed bed. Journal of the Chinese Institute of Chemical Engineers 37, 63-69.

Dwyer, F.P., Mellor, D.P., 1964. Chelating Agents and Metal Chelates. Academic Press, New York, p. 362.

Fournier, M.C., Falk, L., Villermaux, J., 1996. A new parallel competing reaction system for assessing micromixing efficiency-experimental approach. Chemical Engineering Science 51, 5053-5064.

Griffith, E.J., Beeton, A., Spencer, J.M., Mitchell, D.T., 1973. Environmental Phosphorous Handbook, Wiley, New York, pp. 282-286.

Kubota, N., Sekimoto, T., Shimizu, K., 1990. Precipitation of $\mathrm{BaCO}_{3}$ in a semi-batch reactor with double-tube gas injection nozzle. Journal of Crystal Growth 102, 434-440.

Lyons, J.W., 1961. Phosphorus and its Compounds, vol. II. Interscience, New York, pp. 1655-1729.

Ramshaw, C., 1995. The incentive for process intensification. First International Conference of Process Intensification for Chemical Industry, London.

Sondi, I., Matijevic, E., 2003. Homogeneous precipitation by enzymecatalyzed reactions. 2. Strontium and barium carbonates. Chemistry of Materials 15, 1322-1326.

Tagaya, A., Ohkita, H., Mukoh, M., Sakaguchi, R., Koike, Y., 2003. Compensation of the birefringence of a polymer by a birefringent crystal. Science 301, 812-814.

Tai, C.Y., Tai, C.-T., 2005. Development and application of high-gravity crystallization, Technical Report, Research Center for Petrochemical Industry, National Taiwan University, Taipei, Taiwan.

Yagi, H., Suita, Y., Nagashima, S., Hikita, H., 1988. Semibatch precipitation accompanying gas-liquid reaction. Chemical Engineering Communications $65,109-119$. 